\title{
ON THE RESOLUTION OF A CURVE LYING ON A SMOOTH CUBIC SURFACE IN $\mathbb{P}^{3}$
}

\author{
SALVATORE GIUFFRIDA AND RENATO MAGGIONI
}

\begin{abstract}
Let $C$ be any reduced and irreducible curve lying on a smooth cubic surface in $\mathbb{P}^{3}$. In this paper we determine the graded Betti numbers of the ideal sheaf $\mathscr{I}_{C}$.
\end{abstract}

\section{INTRODUCTION}

In this paper we continue the study of curves $C$ on a smooth cubic surface $S$ of $\mathbb{P}^{3}$ begun by the first author in [G and G1].

We obtain a complete description of the graded Betti numbers of the ideal sheaf $\mathscr{I}_{C}$ of $C$ in terms of the seven integers which describe $C$ as a divisor on $S$.

A consequence of our results is that the graded Betti numbers of a reduced and irreducible curve $C$, lying on a smooth cubic (or quadric) surface $S$, do not change within the same linear equivalence class, i.e., they are determined by the class of Pic $S$ to which $C$ belongs. Observe that the starting point of our argument is the knowledge of $\operatorname{Pic} S$ as the group freely generated on a suitable basis.

We would like to bring the reader's attention on the algorithm of Remark 4.7. This was the key tool which first allowed us to understand the behavior of generators and syzygies of a curve on $S$.

As far as we know this kind of problem has been solved, in general, only for arithmetically Cohen-Macaulay curves in $\mathbb{P}^{3}$ : the characterization of the graded Betti numbers for these curves was done in [E], where the work begun in $[\mathrm{PS}]$ is completed.

The content of the various sections is the following: in $\S 1$ we review some basic facts about curves on a smooth cubic and connect the graded Betti numbers of a curve with its Hilbert function.

In $\S 2$ we determine the number of minimal generators of the homogeneous ideal $I(C)$, for each degree $n$, using the intrinsic geometric properties of the curves on $S$.

In the third and fourth sections we find the numbers of the first and second syzygies for each degree $n$, completing in this way the description of the graded Betti numbers of $\mathscr{I}_{C}$. Particular attention is devoted to curves generated in

Received by the editors July 18, 1988 and, in revised form, February 6, 1990.

1980 Mathematics Subject Classification (1985 Revision). Primary 14C20; Secondary 14J25.

Work done with the financial support of the Italian M.P.I. 
degree $\leq h, h+1$, which give some particular problem ( $h$ is the least degree of a surface containing $C$ but not $S$ ).

\section{1}

Let $S$ be a nonsingular cubic surface in $\mathbb{P}^{3}=\mathbb{P}_{k}^{3}$, where $K$ is any algebraically closed field. $S$ is isomorphic to the blow up of $\mathbb{P}^{2}$ in six points $P_{1}, P_{2}, \ldots, P_{6}$ in generic position $[\mathrm{H}, \mathrm{V}, 4]$. If we denote by $L$ the transform of a generic line of $\mathbb{P}^{2}$ and by $E_{i}$ the exceptional curve (which is a line) corresponding to $P_{i}$, then $\operatorname{Pic} S \simeq \mathbb{Z}^{7}$, with free basis $[L],\left[E_{1}\right],\left[E_{2}\right], \ldots,\left[E_{6}\right]$, where $[L],\left[E_{i}\right]$ are the linear equivalence classes of $L, E_{i}$ respectively.

Let $D$ be a divisor on $S$ and let $\mathscr{O}_{S}(D)$ be the corresponding invertible sheaf. We say that $D\left(\right.$ or $\left.\mathscr{O}_{s}(D)\right)$ is of type $\left(a ; b_{1}, b_{2}, \ldots, b_{6}\right)$ if $D \sim a L-\sum_{i=1}^{6} b_{i} E_{i}$.

Throughout this paper a curve is a locally Cohen-Macaulay scheme of pure dimension one; a curve on $S$ is an effective divisor; following $[\mathrm{H}]$ we say that a curve is integral when it is reduced and irreducible.

If $C \subset S$ is a curve of type $\left(a ; b_{1}, b_{2}, \ldots, b_{6}\right)$, then $C$ has degree $d=$ $3 a-\sum_{i=1}^{6} b_{i}$ and arithmetic genus

$$
g=\left(\begin{array}{c}
a-1 \\
2
\end{array}\right)-\sum_{i=1}^{6}\left(\begin{array}{c}
b_{i} \\
2
\end{array}\right) .
$$

If $C^{\prime}$ is another curve on $S$, of type $\left(a^{\prime} ; b_{1}^{\prime}, b_{2}^{\prime}, \ldots, b_{6}^{\prime}\right)$, then the intersection multiplicity is given by

$$
C \cdot C^{\prime}=a a^{\prime}-\sum_{i=1}^{6} b_{i} b_{i}^{\prime},
$$

and the union of the two curves is the divisor of type $\left(a+a^{\prime} ; b_{1}+b_{1}^{\prime}, b_{2}+\right.$ $\left.b_{2}^{\prime}, \ldots, b_{6}+b_{6}^{\prime}\right)$.

We denote by $\pi$ the generic plane section of $S$; this curve is of type $(3 ; 1,1$, $1,1,1,1)$.

If $C \subset S$ is a reduced and irreducible curve of degree $d \geq 3$, of type $\left(a ; b_{1}, b_{2}, \ldots, b_{6}\right)$, with $b_{1} \geq b_{2} \geq \cdots \geq b_{6}$, then the following numerical conditions hold (see [H1, Proposition 2.3]):

$$
\begin{gathered}
a>0, \quad b_{6} \geq 0, \quad a \geq b_{1}+b_{2}, \\
2 a \geq \sum_{i=1}^{5} b_{i}, \quad a^{2}>\sum_{i=1}^{6} b_{i}^{2} .
\end{gathered}
$$

We shall say that a curve $C \subset S$ is of type (*) if it is zero, a line, a conic or, when $d \geq 3$, if the numbers $a, b_{1}, b_{2}, \ldots, b_{6}$ satisfy the conditions (*). In fact a curve $C$ is of type $(*)$ if and only if the generic element of the class $[C]$, to which $C$ belongs, is a smooth connected curve (see [H, p. 407]).

If $C$ is a curve on $S$ we denote by $\mathscr{I}_{C}, \bar{I}_{C}$ the sheaves of ideals of $C$ in $\mathbb{P}^{3}$ and in $S$ respectively, and by $I(C)=\bigoplus_{n>0} H^{0}\left(\mathbb{P}^{3}, \mathscr{I}_{C}(n)\right)$ the homogeneous ideal of $C$ in $K\left[x_{0}, x_{1}, x_{2}, x_{3}\right]$. If $C$ is of type $\left(a ; b_{1}, b_{2}, \ldots, b_{6}\right)$, then $\overline{\mathscr{I}}_{C}$ is of type $\left(-a ;-b_{1},-b_{2}, \ldots,-b_{6}\right)$ and $\mathscr{O}_{s}(C+n \pi)$ is of type $(a+$ $\left.3 n ; b_{1}+n, b_{2}+n, \ldots, b_{6}+n\right)$. For every sheaf $\mathscr{F}$ on a scheme $X$ we put, as usual, $h^{i}(X, \mathscr{F})=\operatorname{dim}_{k} H^{i}(X, \mathscr{F})$. 
Let $C \subset S$ be a curve of type $(*)$; we denote by $H(C,-)$ its Hilbert function and by $P(C,-)$ its Hilbert polynomial. If $C$ is an integral curve, then $H(C,-)$ has been calculated in [G1]; we shall see that $H(C,-)$ is independent of the choice of $C$ in its linear equivalence class (see below, Remark 2.5).

Definition 1.1. Let $C \subset S$ be a curve of type $(*)$. We put

$$
h=\min \left\{n: H^{0}\left(S, \overline{\mathscr{I}}_{C}(n)\right) \neq 0\right\},
$$

i.e., $h$ is the least degree of a surface containing $C$ but not $S$.

For any $n \geq h$ we denote by $C_{n}^{\prime}$ the curve linked to $C$ in the complete intersection of $S$ with a surface of degree $n$ containing $C$ but not $S$. We denote by $k$ the minimum degree $n$ such that $C_{n}^{\prime}$ is a reduced curve.

Observe that there exists a natural isomorphism $\overline{\mathscr{I}}_{C}(n) \simeq \mathscr{O}_{S}\left(C_{n}^{\prime}\right)$; in fact $\overline{\mathscr{I}}_{C} \simeq \mathscr{O}_{S}(-C)$, and $\mathscr{O}_{S}\left(C_{n}^{\prime}\right) \simeq \mathscr{O}_{S}(n \pi-C)$.

Recall that, for $n \geq k, H(C, n)=P(C, n)$ (see [G1, Theorem 1.4]) and that, for a given curve $C \subset S$ of type $(*)$, the numbers $h$ and $k$ can be determined by simple algorithms. To determine $h$ we can alternatively use the algorithm of Harbourne (see [Ha]), or that of Remark 1.2 in [G1]. The number $k$ can be determined even in a simpler way: if $C$ is of type $\left(a ; b_{1}, b_{2}, \ldots, b_{6}\right)$ with $b_{1} \geq b_{2} \geq \cdots \geq b_{6}$, then (see [G1, p. 278])

$k=\min \left\{m: m \geq h ; m \geq b_{1}-1 ; m \geq a-b_{5}-b_{6}-1 ; m \geq 2 a-\sum_{i=2}^{6} b_{i}-1\right\}$.

In the sequel we suppose, for curves on $S$, that $h \geq 3$, i.e., $C$ is not contained on a quadric surface (except for Theorem 4.8).

In this paper we use the sequences $\Delta^{i} H(C,-)$ of the $i$ th differences of the Hilbert function, which are defined in the following way: for each $n \geq 0$ we put

$$
\begin{gathered}
\Delta H(C, n)=H(C, n)-H(C, n-1), \\
\Delta^{i} H(C, n)=\Delta\left(\Delta^{i-1} H(C, n)\right) .
\end{gathered}
$$

In particular we shall deal with the sequence $\Delta^{4} H(C,-)$.

For each curve $C \subset \mathbb{P}^{3}$ its ideal sheaf $\mathscr{I}_{C}$ has a minimal free resolution with morphisms of degree zero:

$$
0 \rightarrow \bigoplus_{i=1}^{p} \mathscr{O}\left(-c_{i}\right) \rightarrow \bigoplus_{i=1}^{q} \mathscr{O}\left(-b_{i}\right) \rightarrow \bigoplus_{i=1}^{r} \mathscr{O}\left(-a_{i}\right) \rightarrow \mathscr{I}_{C} \rightarrow 0,
$$

where $\mathscr{O}=\mathscr{O}_{\mathbb{P}^{3}}$; this notation will be used throughout the paper. Here $r$ is the number of elements of a minimal set of generators of the homogeneous ideal $I(C), a_{i}$ are the degrees of these generators; $q$ (resp. $p$ ) is the number of a minimal set of generators of the module of first (resp. second) syzygies and $b_{i}$ (resp. $c_{i}$ ) are their degrees.

We assume $a_{1} \leq a_{2} \leq \cdots \leq a_{r} ; b_{1} \leq b_{2} \leq \cdots \leq b_{q} ; c_{1} \leq c_{2} \leq \cdots \leq c_{p}$, and for each $n$ we put

$$
\alpha_{n}=\#\left\{a_{i}: a_{i}=n\right\}, \quad \beta_{n}=\#\left\{b_{i}: b_{i}=n\right\}, \quad \gamma_{n}=\#\left\{c_{i}: c_{i}=n\right\} .
$$


The integers $\alpha_{n}, \beta_{n}, \gamma_{n}$ are the graded Betti numbers of the ideal sheaf $\mathscr{I}_{C}$. From the above resolution one can compute the following numbers: $\sigma=$ $\max \left\{n: H^{1}\left(\mathbb{P}^{3}, \mathscr{I}_{C}(n)\right) \neq 0\right\}, \varepsilon=e(C)=\max \left\{n: H^{2}\left(\mathbb{P}^{3}, \mathscr{I}_{C}(n)\right) \neq 0\right\}$.

A curve $C \subset \mathbb{P}^{3}$ is arithmetically Cohen-Macaulay (shortly ACM) if and only if $p=0$; the resolutions of such curves are given by Ellingsrud in [E]. The graded Betti numbers of ACM curves having assigned Hilbert function have been studied by several authors [GP, C, MR1], and in [S] the graded Betti numbers of smooth ACM curves are characterized; a general survey on the relations among the results of these authors is given in [GeMi1].

The graded Betti numbers of ACM curves lying on a smooth quadric are well known (see e.g. [G1, §6]); the same problem on a smooth cubic surface is solved in [W, G1, and P] by different methods. In particular in [G1] it is shown that the graded Betti numbers of an ACM curve $C$ lying on a smooth cubic depend only on the degree and the genus of $C$; hence they can be read in an explicit way from the sequence $\Delta^{4} H(C,-)$ (see (5) of the next lemma). In fact, for these curves there are no generators and syzygies in the same degree.

Lemma 1.2. Let $C \subset \mathbb{P}^{3}$ be a non-ACM curve. With the above notation we have:

(1) $p+r=q+1$, and $-\sum_{i=1}^{r} a_{i}+\sum_{i=1}^{q} b_{i}-\sum_{i=1}^{p} c_{i}=0$,

(2) $a_{2}<b_{1}, b_{2}<c_{1}, b_{q}>a_{r}$,

(3) if $\sigma>\varepsilon$, then $c_{p}>b_{q}$,

(4) for every $n \geq 0$,

$$
H(C, n)=\left(\begin{array}{c}
n+3 \\
3
\end{array}\right)-\sum_{i=0}^{n}\left(\alpha_{i}-\beta_{i}+\gamma_{i}\right)\left(\begin{array}{c}
n-i+3 \\
3
\end{array}\right)
$$

(5) for every $n>0, \Delta^{4} H(C, n)=-\alpha_{n}+\beta_{n}-\gamma_{n}$.

Proof. Items (1) and (2) are standard.

(3) (Ph. Ellia). We prove that $b_{q} \geq c_{p}$ implies $\sigma \leq \varepsilon$. Splitting the resolution of $\mathscr{I}_{C}$ we get

$$
\begin{gathered}
0 \rightarrow \bigoplus_{i=1}^{p} \mathscr{O}\left(-c_{i}\right) \rightarrow \bigoplus_{i=1}^{q} \mathscr{O}\left(-b_{i}\right) \rightarrow \mathscr{E} \rightarrow 0, \\
0 \rightarrow \mathscr{E} \rightarrow \bigoplus_{i=1}^{r} \mathscr{O}\left(-a_{i}\right) \rightarrow \mathscr{I}_{C} \rightarrow 0
\end{gathered}
$$

where $\mathscr{E}$ is a locally free sheaf.

From these short exact sequences, tensoring with $\mathscr{O}(n)$ for suitable $n$, taking cohomology and applying Serre duality, we have

$$
\begin{aligned}
H^{2}\left(\mathbb{P}^{3}, \mathscr{E}(n)\right) & \simeq H^{1}\left(\mathbb{P}^{3}, \mathscr{I}_{C}(n)\right) \text { for any } n ; \\
0 \rightarrow H^{2}\left(\mathbb{P}^{3}, \mathscr{E}(n)\right) & \rightarrow H^{3}\left(\mathbb{P}^{3}, \bigoplus_{i=1}^{p} \mathscr{O}\left(-c_{i}+n\right)\right) \rightarrow \cdots
\end{aligned}
$$

thus we see that $H^{1}\left(\mathbb{P}^{3}, \mathscr{I}_{C}(n)\right)=0$ for any $n>c_{p}-4$. This implies $\sigma \leq c_{p}-4$.

From (i) we get

$$
\begin{aligned}
\cdots \rightarrow H^{3}\left(\mathbb{P}^{3}, \bigoplus_{i=1}^{p} \mathscr{O}\left(-c_{i}+b_{q}-4\right)\right) & \stackrel{f}{\rightarrow} H^{3}\left(\mathbb{P}^{3}, \bigoplus_{i=1}^{q} \mathscr{O}\left(-b_{i}+b_{q}-4\right)\right) \\
& \rightarrow H^{3}\left(\mathbb{P}^{3}, \mathscr{E}\left(b_{q}-4\right)\right) \rightarrow 0 .
\end{aligned}
$$


Since the resolution of $\mathscr{I}_{C}$ is minimal, whether $c_{p}<b_{q}$ or $c_{p}=b_{q}$ the matrix of $f$ must have at least one row of zeros, hence the map $f$ is not surjective, and $H^{3}\left(\mathbb{P}^{3}, \mathscr{E}\left(b_{q}-4\right)\right) \neq 0$.

Similarly, from (ii)

$$
\begin{aligned}
0 \rightarrow & H^{2}\left(\mathbb{P}^{3}, \mathscr{I}_{C}\left(b_{q}-4\right)\right) \rightarrow H^{3}\left(\mathbb{P}^{3}, \mathscr{E}\left(b_{q}-4\right)\right) \\
& \rightarrow H^{3}\left(\mathbb{P}^{3}, \bigoplus_{i=1}^{r} \mathscr{O}\left(-a_{i}+b_{q}-4\right)\right) \rightarrow \cdots
\end{aligned}
$$

Now, since $H^{3}\left(\mathbb{P}^{3}, \bigoplus_{i=1}^{r} \mathscr{O}\left(-a_{i}+b_{q}-4\right)\right)=0$ (see item (2)), we conclude that $\varepsilon \geq b_{q}-4 \geq c_{p}-4 \geq \sigma$.

For item (4) we have

$$
H(C, n)=\left(\begin{array}{c}
n+3 \\
3
\end{array}\right)-\sum_{i=1}^{r}\left(\begin{array}{c}
n-a_{i}+3 \\
3
\end{array}\right)+\sum_{i=1}^{q}\left(\begin{array}{c}
n-b_{i}+3 \\
3
\end{array}\right)-\sum_{i=1}^{p}\left(\begin{array}{c}
n-c_{i}+3 \\
3
\end{array}\right),
$$

where we assume that $\left(\begin{array}{c}m \\ 3\end{array}\right)=0$ for $m<3$. The assertion follows by definition of $\alpha_{i}, \beta_{i}, \gamma_{i}$.

The proof of (5) needs explicit computation; the first step is

$$
\begin{aligned}
\Delta H(C, n) & =\left(\begin{array}{c}
n+2 \\
2
\end{array}\right)-\sum_{i=0}^{n-1}\left(\alpha_{i}-\beta_{i}+\gamma_{i}\right)\left(\begin{array}{c}
n-i+2 \\
2
\end{array}\right)-\left(\alpha_{n}-\beta_{n}+\gamma_{n}\right) \\
& =\left(\begin{array}{c}
n+2 \\
2
\end{array}\right)-\sum_{i=0}^{n}\left(\alpha_{i}-\beta_{i}+\gamma_{i}\right)\left(\begin{array}{c}
n-i+2 \\
2
\end{array}\right) \cdot
\end{aligned}
$$

Remark 1.3. When $C$ is an ACM curve, it is easy to see that (1), (2), (4), (5) hold, making the necessary changes.

Lemma 1.4. Let $C \subset S$ be a curve of type (*) which is not ACM and does not lie on a quadric. Then $\sigma>\varepsilon$.

Proof. First observe that $\varepsilon<h-1$ since $C$ is not a complete intersection; if $\sigma \geq h-1$ we are done, so we can assume that $\sigma<h-1$. We show that $h^{1}\left(\mathbb{P}^{3}, \mathscr{I}_{C}(h-2)\right)>0$ and $h^{2}\left(\mathbb{P}^{3}, \mathscr{I}_{C}(h-2)\right)=0$. By the previous assumptions $\Delta H(C, h)=d(d=\operatorname{deg} C)$, and since $C$ is not ACM we have $\Delta H(C, h-1)>d$ (see [HE, Remark 3.1.1]). Thus $H(C, h-1)-H(C, h-2)=$ $P(C, h-1)-P(C, h-2)+h^{1}\left(\mathbb{P}^{3}, \mathscr{I}_{C}(h-2)\right)-h^{2}\left(\mathbb{P}^{3}, \mathscr{I}_{C}(h-2)\right)>d$; hence we have

$$
h^{1}\left(\mathbb{P}^{3}, \mathscr{I}_{C}(h-2)\right)-h^{2}\left(\mathbb{P}^{3}, \mathscr{I}_{C}(h-2)\right)>0 \text {. }
$$

Now we use the formula [MR, Applications]

$$
h^{2}\left(\mathbb{P}^{3}, \mathscr{I}_{C}(n)\right)=H(X, \bar{n})-H\left(C_{h}^{\prime}, \bar{n}\right),
$$

where $C, C_{h}^{\prime}$ are linked in the complete intersection $X$ of $S$ with a surface of degree $h, \bar{n}=h-1-n$. Setting $n=h-2$ we conclude

$$
h^{2}\left(\mathbb{P}^{3}, \mathscr{I}_{C}(h-2)\right)=H(X, 1)-H\left(C_{h}^{\prime}, 1\right)=0,
$$

because $C_{h}^{\prime}$ is not a plane curve.

By Lemmas 1.2 and 1.4, for non-ACM curves of type $(*)$ on a smooth cubic surface we always have $c_{p}>b_{q}$. This fact is frequently used in $\S \S 3$ and 4 . The 
same is true for non-ACM curves lying on a smooth quadric, i.e., for curves of type $(a, b)$ with $1 \leq a<b-1$ [GM, Theorem 3.10].

\section{2}

Let $C \subset S$ be a curve of type $(*)$, not contained on quadric surface (see Theorem 4.8 for curves on a quadric). In this section we find the number $\alpha_{n}$ of minimal generators of the homogeneous ideal $I(C)$, for each degree $n$. Clearly $\alpha_{n}=0$ for $n<h, n \neq 3$, and of course $\alpha_{3} \geq 1$ because of $S$.

Theorem 2.1. Let $C \subset \mathbb{P}^{3}$ be an integral nondegenerate curve, $s$ the minimum degree of a surface $S$ containing $C$, and let $k$ be the minimum degree of $a$ surface $T$ containing $C$ but not $S$, such that $C^{\prime}$, linked to $C$ in $S \cap T$, is a reduced curve.

Then the homogeneous ideal $I(C)$ is generated in degree $\leq s+k-2$.

Proof. We need only to show that the ideal sheaf $\mathscr{I}_{C}$ is $(s+k-2)$-regular (see [M, lecture 14]). From the exact sequence

$$
0 \rightarrow \mathscr{I}_{S \cap T}(n) \rightarrow \mathscr{I}_{C}(n) \rightarrow \omega_{C^{\prime}}(n-e+4) \rightarrow 0,
$$

where $\omega_{C^{\prime}}$ is the dualizing sheaf of $C^{\prime}, e=k+s$, we get

$$
0 \rightarrow H^{1}\left(\mathbb{P}^{3}, \mathscr{I}_{C}(n)\right) \rightarrow H^{1}\left(C^{\prime}, \omega_{C^{\prime}}(n-e+4)\right) \rightarrow \cdots
$$

By duality

$$
H^{1}\left(C^{\prime}, \omega_{C^{\prime}}(n-e+4)\right) \simeq H^{0}\left(C^{\prime}, \mathscr{O}_{C^{\prime}}(e-n-4)\right)^{\vee},
$$

and the last group vanishes when $e-n-4<0$, i.e., $n \geq s+k-3$, since $C^{\prime}$ is a reduced curve. Hence $H^{1}\left(\mathbb{P}^{3}, \mathscr{I}_{C}(n)\right)=0$ for $n \geq s+k-3$.

Now, from the exact sequence

$$
0 \rightarrow \mathscr{I}_{C}(n) \rightarrow \mathscr{O}(n) \rightarrow \mathscr{O}_{C}(n) \rightarrow 0,
$$

we get

$$
H^{2}\left(\mathbb{P}^{3}, \mathscr{I}_{C}(s+k-4)\right) \simeq H^{1}\left(C, \mathscr{O}_{C}(s+k-4)\right)=0,
$$

by the speciality theorem (see [GP, $§ 1]$ ) (if $C=S \cap T$, the theorem is trivially true).

Finally $H^{3}\left(\mathbb{P}^{3}, \mathscr{I}_{C}(s+k-5)\right) \simeq H^{3}\left(\mathbb{P}^{3}, \mathscr{O}(s+k-5)\right)=0$.

When $s=3$, the ideal sheaf $\mathscr{I}_{C}$ is $(k+1)$-regular, so $I(C)$ is generated in degree $\leq k+1$, and $\alpha_{n}=0$ for $n>k+1$.

We shall need the following lemma.

Lemma 2.2. Let $C \subset S$ be a curve of type (*) or composed with a pencil, and let $L \subset S$ be a line intersecting $C$. Then $C+L$ is a curve of type (*).

Proof. If $C=0$ or $C$ is a line, the lemma is true. Let $\left(a ; b_{1}, b_{2}, \ldots, b_{6}\right)$ be the type of $C$; suppose that $L$ is, say, of type $(2 ; 1,1,1,1,1,0)$. We must check that the 7-tuple $\left(a+2 ; b_{1}+1, \ldots, b_{5}+1, b_{6}\right)$ satisfies conditions $(*)$. The only nontrivial checks are the following:

$$
2(a+2) \geq \sum_{i=1}^{5}\left(b_{i}+1\right), \quad(a+2)^{2}>\sum_{i=1}^{5}\left(b_{i}+1\right)^{2}+b_{6}^{2} .
$$


The first inequality is $2 a-\sum_{i=1}^{5} b_{i} \geq 1$, and this is true because $L$ is a secant for $C$; the second one is true because the hypothesis on $C$ gives $a^{2} \geq \sum_{i=1}^{6} b_{i}^{2}$ (see [G, p. 255]), and $2\left(2 a-\sum_{i=1}^{5} b_{i}\right)>1$ because $L$ intersects $C$.

For lines of different types one can use similar arguments.

Let $D$ be an effective divisor on a smooth cubic surface, of type $\left(a ; b_{1}, b_{2}\right.$, $\left.\ldots, b_{6}\right)$; we want to determine $h^{0}\left(S, \mathscr{O}_{S}(D)\right)$. This dimension is already known (see [G1]); but we want to show the technique that will be used because it is interesting in its own right.

Throughout the paper we shall say that a curve $D^{\prime}$ is a fixed component of the curve $D$ if $D^{\prime}$ is a fixed component of the complete linear system $|D|$.

We say that a curve $C \subset S$ is isolated if $h^{0}\left(S, \mathscr{O}_{S}(C)\right)=1$, i.e., if $C$ is not linearly equivalent to any other curve on $S$. By Corollary 1.3 and Theorem 1.4 of [G] we see that the integral isolated curves on $S$ are the 27 lines of $S$ (recall that for each of these lines $L$ one has $L^{2}=-1$ ); hence for any effective divisor $D \subset S$ the integral fixed components of $D$ are necessarily lines. Now we want to see how to check when a line $L$ is a fixed component of $D$ : this happens when $L \cdot D=-s<0$; in this case $L \cdot(D-L)=-s+1$. Hence the line of type $(1 ; 1,1,0,0,0,0)$ is a fixed component of $D$, with multiplicity $s$, if $s=b_{1}+b_{2}-a>0$; the line of type $(2 ; 1,1,1,1,1,0)$ is a fixed component of $D$ with multiplicity $s$, if $s=\sum_{i=1}^{5} b_{i}-2 a>0$; the line of type $(0 ;-1,0,0,0,0,0)$ is a fixed component of $D$ with multiplicity $s$, if $s=-b_{1}>0$. The same is true for the other 24 lines, since they are obtained from the previous three by permutations.

We add to Lemma 2.2 the following remark: if $C \subset S$ is any curve and $L$ is a line on $S$ such that $C \cdot L=0$, then $L$ is a fixed component of $|C+L|$ : in fact we have $L \cdot(C+L)=L^{2}=-1$.

By subtracting from $D$ all its fixed components, say $L_{1}, L_{2}, \ldots, L_{p}$, each with its own multiplicity $s_{1}, s_{2}, \ldots, s_{p}$, we get

$$
D=s_{1} L_{1}+s_{2} L_{2}+\cdots+s_{p} L_{p}+\bar{D},
$$

where either $\bar{D}$ is a curve of type $(*)$ (but not a line) or it is composed with a pencil of conics [G, §2]. In any case, if $\bar{D}$ is of type $\left(\bar{a} ; \bar{b}_{1}, \bar{b}_{2}, \ldots, \bar{b}_{6}\right)$, one has $[\mathrm{G}$, Theorem 1.4]

$$
h^{0}\left(S, \mathscr{O}_{S}(D)\right)=h^{0}\left(S, \mathscr{O}_{S}(\bar{D})\right)=\left(\begin{array}{c}
\bar{a}+2 \\
2
\end{array}\right)-\sum_{i=1}^{6}\left(\begin{array}{c}
\bar{b}_{i}+1 \\
2
\end{array}\right) .
$$

Recall that one can decide if a curve $\bar{D}$, free from fixed components, is composed with a pencil: this happens if and only if $\bar{D}^{2}=0$ (see [G, proof of Theorem 2.5]).

Remark 2.3. We say that (1) is the Zariski decomposition of $D[\mathrm{Z}, \S 7]$; for it the following relevant facts hold:

(i) $L_{i} \cdot L_{j}=0 \quad(i, j=1,2, \ldots, p ; i \neq j)$ i.e., the distinct lines which form the fixed components of $|D|$ are mutually skew. This is easily seen: if two of these lines were not skew, say $L_{i} \cdot L_{j}=1$, then $L_{i}+L_{j}$ would be linearly equivalent to a conic, hence it would move in a pencil (see [G1, Proposition 
2.2]). In this case the linear system $\left|L_{i}+L_{j}+\bar{D}\right|$ might not have fixed components. A consequence of this fact is that $p \leq 6$ : in fact it is well known that on a smooth cubic surface there exist at most six mutually skew lines.

(ii) $L_{i} \cdot \bar{D}=0(i=1,2, \ldots, p)$ i.e., the lines which form the fixed component of $D$ are skew with the "moving component" of $D$. In fact, if $L_{i} \cdot \bar{D}>0$, then the divisor $L_{i}+\bar{D}$ is of type $(*)$ by Lemma 2.2 , so the linear system $\left|L_{i}+\bar{D}\right|$ has no fixed components.

(iii) Observe that $h^{1}\left(S, \mathscr{O}_{S}(D)\right)=h^{1}\left(S, \mathscr{I}_{C}(n)\right)$, where $C$ is an integral curve linked to $D$ by a complete intersection of $S$ with a surface of degree $n$ large enough; hence the superabundance of $|D|$ is given by: $h^{1}\left(S, \mathscr{O}_{S}(D)\right)=$ $\sum_{i=1}^{p}\left(\begin{array}{c}s_{i} \\ 2\end{array}\right)$ (see [G1, Theorem 2.4]). Note that if $D$ is reduced it has no superabundance.

Remark 2.4. Let $C \subset S$ be a curve of type $(*)$; we shall often deal with the Zariski decomposition of the linked curves $C_{n}^{\prime}$ (see Definition 1.1). It is easy to verify that if $C_{n}^{\prime}=s_{1} L_{1}+s_{2} L_{2}+\cdots+s_{p} L_{p}+\bar{C}_{n}$ then $C_{n+1}^{\prime}=$ $\left(s_{1}-1\right) L_{1}+\left(s_{2}-1\right) L_{2}+\cdots+\left(s_{p}-1\right) L_{p}+\bar{C}_{n+1}$, that is, the fixed components of $C_{n+1}^{\prime}$ are those of $C_{n}^{\prime}$, each with multiplicity decreased exactly by one (if $s_{i}=1$, of course, $L_{i}$ is not a fixed component of $\left.C_{n+1}^{\prime}\right)$. Indeed if $C$ is of type $\left(a ; b_{1}, b_{2}, \ldots, b_{6}\right)$, then $C_{n}^{\prime}$ is of type $\left(3 n-a ; n-b_{1}, n-b_{2}, \ldots, n-b_{6}\right)$, and $C_{n+1}^{\prime}$ is of type $\left(3 n+3-a ; n+1-b_{1}, n+1-b_{2}, \ldots, n+1-b_{6}\right)$. If $L_{1}$ is of type $(1 ; 1,1,0,0,0,0)$, then $s_{1}=\left(n-b_{1}\right)+\left(n-b_{2}\right)-(3 n-a)=a-b_{1}-b_{2}-n>0$, and $\left(n+1-b_{1}\right)+\left(n+1-b_{2}\right)-(3 n+3-a)=a-b_{1}-b_{2}-n-1=s_{1}-1$.

A similar computation can be done for any other line.

Remark 2.5. By the above argument one can directly compute the Hilbert function of $C$. Indeed from the exact sequence

$$
0 \rightarrow \mathscr{I}_{S}(n) \rightarrow \mathscr{I}_{C}(n) \rightarrow \overline{\mathscr{I}}_{C}(n) \rightarrow 0,
$$

where $\mathscr{I}_{S}$ is the ideal sheaf of $S$ in $\mathbb{P}^{3}$, one has, for any $n \geq 0$ :

so

$$
h^{0}\left(\mathbb{P}^{3}, \mathscr{I}_{C}(n)\right)=h^{0}\left(S, \overline{\mathscr{I}}_{C}(n)\right)+\left(\begin{array}{c}
n \\
3
\end{array}\right)
$$

$$
\begin{aligned}
H(C, n) & =\left(\begin{array}{c}
n+3 \\
3
\end{array}\right)-h^{0}\left(\mathbb{P}^{3}, \mathscr{I}_{C}(n)\right) \\
& =3\left(\begin{array}{c}
n+1 \\
2
\end{array}\right)+1-h^{0}\left(S, \mathscr{O}_{S}\left(\bar{C}_{n}\right)\right) .
\end{aligned}
$$

This shows that the Hilbert function of a curve $C \subset S$ depends only on the equivalence class to which the curve belongs; that is, on the seven numbers $\left(a ; b_{1}, b_{2}, \ldots, b_{6}\right)$.

Moreover, since passing from $C_{n}^{\prime}$ to $C_{n+1}^{\prime}$ decreases the multiplicity of the fixed lines exactly by one, the knowledge of the Zariski decomposition of $C_{h}^{\prime}$ allows us to determine the number $k$ (see Definition 1.1); namely, if $C_{h}^{\prime}=$ $s_{1} L_{1}+s_{2} L_{2}+\cdots+s_{p} L_{p}+\bar{C}_{h}\left(s_{1} \geq s_{2} \geq \cdots \geq s_{p}\right)$, then $k=h+s_{1}-1$ when $s_{1}>1, k=h$ otherwise. In fact if $s_{1}>1$ the curve $C_{h+s_{1}-1}^{\prime}$ is reduced, while $C_{h+s_{1}-2}^{\prime}$ is not; if $s_{1}=0$ or $s_{1}=1$ of course $h=k$. Then the algorithm used to calculate the multiplicities $s_{i}$, together with the above formula, can substitute for the algorithm (given after Definition 1.1) to find $k$. 
Proposition 2.6. Let $C \subset S$ be a curve of type (*). A line $L$ is a fixed component of $C_{n}^{\prime}$, with multiplicity $s$, if and only if $L$ is a $(n+s)$-secant of $C$.

Proof. Assume that $C$ is of type $\left(a ; b_{1}, b_{2}, \ldots, b_{6}\right)$, so $C_{n}^{\prime}$ is of type $(3 n-$ $\left.a ; n-b_{1}, n-b_{2}, \ldots, n-b_{6}\right)$. The line $(1 ; 1,1,0,0,0,0)$ is a fixed component of $C_{n}^{\prime}$, of multiplicity $s$, iff $\left(n-b_{1}\right)+\left(n-b_{2}\right)-(3 n-a)=s$, that is iff $a-b_{1}-b_{2}=n+s$, that is iff the line is a $(n+s)$-secant of $C$. The same argument holds for any other line on $S$.

The following result has an important role in the next theorem.

Lemma 2.7. Let $C \subset S$ be a curve without fixed components. Then the cupproduct morphism

$$
\varphi: H^{0}\left(S, \mathscr{O}_{S}(C)\right) \otimes H^{0}\left(S, \mathscr{O}_{S}(1)\right) \rightarrow H^{0}\left(\mathscr{O}_{S}(C+\pi)\right)
$$

is surjective.

Proof. If $C$ is of type (*) the conclusion follows by [GM, Lemma 3.2]. So we can suppose that $C$ is composed with a pencil of conics; we know that it is cut out on $S$ by a pencil of planes having for axis a line $L \subset S$. Call $\Gamma$ a conic of the pencil and let $C=\lambda \Gamma, \lambda>1:$ then $\operatorname{deg} C=2 \lambda, h^{0}\left(S, \mathscr{O}_{S}(C)\right)=\lambda+1$, $h^{0}\left(S, \mathscr{O}_{S}(C+\pi)\right)=3 \lambda+4$, as one can compute starting with any conic (e.g. $\Gamma$ of type $(1 ; 1,0,0,0,0,0))$. Following the proof of the quoted lemma we shall produce $3 \lambda+4$ independent elements in $\operatorname{Im} \varphi$.

Let $C_{1}, C_{2}, \ldots, C_{\lambda+1}$ be a basis of $H^{0}\left(S, \mathscr{O}_{S}(C)\right)$; we prove that the following elements are linearly independent:

$$
\begin{array}{ll}
C_{1} \pi_{1}, C_{2} \pi_{1}, \ldots, C_{\lambda+1} \pi_{1}, & \lambda+1 \text { elements, } \\
C_{1} \pi_{2}, C_{2} \pi_{2}, \ldots, C_{\lambda+1} \pi_{2}, & \lambda+1 \text { elements, } \\
C_{1} \pi_{3}, C_{2} \pi_{3}, \ldots, C_{\lambda+1} \pi_{3}, & \lambda+1 \text { elements, } \\
C_{1} \pi_{4}, & 1 \text { element. }
\end{array}
$$

Here we choose $\pi_{1}, \pi_{2}$ such that $\pi_{1} \cap \pi_{2}=L^{\prime} \subset S$ is skew with $L, C_{1}$ does not vanish at $\pi_{1} \cap \pi_{2} \cap \pi_{3}=\{P\}$, and $P \notin \pi_{4}$. The elements in the first two rows are independent; $C_{1} \pi_{4}$ does not vanish at $P$, while all the others do. Finally, any element $\left(\alpha_{1} C_{1}+\cdots+\alpha_{\lambda+1} C_{\lambda+1}\right) \pi_{3}, \alpha_{i} \in K$, vanishes at the generic point of $L^{\prime}$ only when $\alpha_{i}=0$ since no element of $|C|$ can contain $L^{\prime}$; the conclusion now follows.

Theorem 2.8. Let $C \subset S$ be a curve of type (*) not lying on a quadric. Then the generators of $I(C)$, besides $S$, are in number:

$$
\alpha_{h}=h^{0}\left(S, \mathscr{O}_{S}\left(\bar{C}_{h}\right)\right),
$$

and for any $n>h$

$$
\alpha_{n}=h^{0}\left(S, \mathscr{O}_{S}\left(\bar{C}_{n}\right)\right)-h^{0}\left(S, \mathscr{O}_{S}\left(\bar{C}_{n-1}+\pi\right)\right),
$$

where $\pi$ is the generic plane section of $S$. 
Proof. From the commutative diagram with exact columns

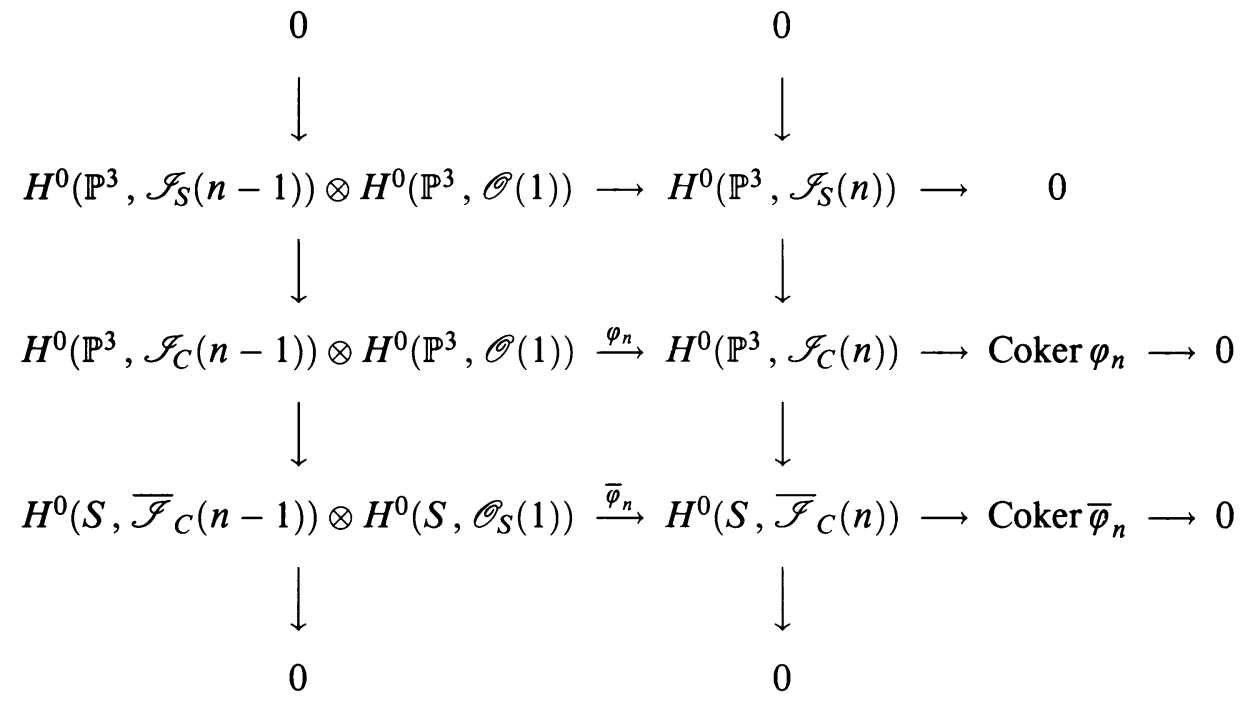

by the snake lemma we get $\operatorname{Coker} \varphi_{n} \simeq \operatorname{Coker} \bar{\varphi}_{n}$. Hence $\alpha_{n}=\operatorname{dim} \operatorname{Coker} \bar{\varphi}_{n}$; to find this number we need to know $\operatorname{dim} \operatorname{Im} \bar{\varphi}_{n}$.

Suppose $n>h$ and put $C_{n-1}^{\prime}=s_{1} L_{1}+s_{2} L_{2}+\cdots+s_{p} L_{p}+\bar{C}_{n-1}$, with $s_{1} \geq s_{2} \geq \cdots \geq s_{p}>0$. Since $\overline{\mathscr{I}}_{C}(n) \simeq \mathscr{O}_{S}\left(C_{n}^{\prime}\right)$, we can write

$$
\bar{\varphi}_{n}: H^{0}\left(S, \mathscr{O}_{S}\left(C_{n-1}^{\prime}\right)\right) \otimes H^{0}\left(S, \mathscr{O}_{S}(1)\right) \rightarrow H^{0}\left(S, \mathscr{O}_{S}\left(C_{n}^{\prime}\right)\right) \text {. }
$$

It is clear that $\operatorname{Im} \bar{\varphi}_{n}$ contains $\sum_{i=1}^{p} s_{i} L_{i}$ in its fixed part, so

$$
\operatorname{Im} \bar{\varphi}_{n} \subset H^{0}\left(S, \mathscr{O}_{S}\left(C_{n}^{\prime}-\sum_{i=1}^{p} L_{i}\right)\right) \text {, }
$$

hence we may consider the map

$$
\psi: H^{0}\left(S, \mathscr{O}_{S}\left(\bar{C}_{n-1}\right)\right) \otimes H^{0}\left(S, \mathscr{O}_{S}(1)\right) \rightarrow H^{0}\left(S, \mathscr{O}_{S}\left(\bar{C}_{n}-\sum_{i=1}^{p} L_{i}\right)\right)
$$

On the other hand we have on $S$ the equivalence $\bar{C}_{n}-\sum_{i=1}^{p} L_{i} \sim \bar{C}_{n-1}+\pi$ so that we can apply Lemma 2.7 to $\psi$. Now the conclusion follows since

$$
\begin{aligned}
\operatorname{dim} \operatorname{Im} \bar{\varphi}_{n} & =\operatorname{dim} \operatorname{Im} \psi=h^{0}\left(S, \mathscr{O}_{S}\left(\bar{C}_{n}-L_{1}-L_{2}-\cdots-L_{p}\right)\right) \\
& =h^{0}\left(S, \mathscr{O}_{S}\left(\bar{C}_{n-1}+\pi\right)\right) .
\end{aligned}
$$

In the case $n=h$ one has

$$
\operatorname{Coker} \bar{\varphi}_{n} \simeq H^{0}\left(S, \overline{\mathscr{I}}_{C}(h)\right),
$$

hence $\alpha_{h}=h^{0}\left(S, \mathscr{O}_{S}\left(\bar{C}_{h}\right)\right)$.

Lemma 2.9. Let $D \subset S$ be a curve, and let $L \subset S$ be a line meeting $D$ in $l$ points, $l \geq 0$. If $D-L$ is a reduced curve, then

$$
h^{0}\left(S, \mathscr{O}_{S}(D-L)\right)=h^{0}\left(S, \mathscr{O}_{S}(D)\right)-1-l .
$$

Proof. Consider the exact sequence

$$
0 \rightarrow \mathscr{O}_{S}(D-L) \rightarrow \mathscr{O}_{S}(D) \rightarrow \mathscr{O}_{L}(D) \rightarrow 0,
$$


and observe that $h^{0}\left(S, \mathscr{O}_{L}(D)\right)=l+1$, and $h^{1}\left(S, \mathscr{O}_{S}(D-L)\right)=0$. The last assertion is true because $D-L$ is a reduced curve (item (iii) of Remark 2.3). Now the lemma follows taking cohomology.

Theorem 2.10. Let $C \subset S$ be a curve of type (*). For $n>h$ if $C_{n-1}^{\prime}=$ $s_{1} L_{1}+s_{2} L_{2}+\cdots+s_{p} L_{p}+\bar{C}_{n-1}$, then $\alpha_{n}=p$.

Proof. We have already seen that, for $n>h$,

$$
\alpha_{n}=h^{0}\left(S, \mathscr{O}_{S}\left(\bar{C}_{n}\right)\right)-h^{0}\left(S, \mathscr{O}_{S}\left(\bar{C}_{n}-L_{1}-L_{2}-\cdots-L_{p}\right)\right)
$$

where the lines $L_{1}, L_{2}, \ldots, L_{p}$ are the fixed distinct components of $C_{n-1}^{\prime}$. We have two cases.

(1) $L_{i} \quad(i=1,2, \ldots, p)$ is a fixed component of $C_{n}^{\prime}$ : in this case it is a zero-secant for $\bar{C}_{n}$ (see Remark 2.3(ii)).

(2) $L_{i} \quad(i=1,2, \ldots, p)$ is not a fixed component of $C_{n}^{\prime}$ : then $L_{i}$ is a fixed simple line of $C_{n-1}^{\prime}$, hence it is a zero-secant for $C_{n}^{\prime}$ and for $\bar{C}_{n}$ : in fact $L_{i} \cdot C_{n}^{\prime}=L_{i} \cdot\left(C_{n-1}^{\prime}+\pi\right)=-1+1=0$.

In any case, we can apply Lemma 2.9 since $\bar{C}_{n}-L_{1}-\cdots-L_{i} \sim \bar{C}_{n-1}+\pi+$ $L_{i+1}+\cdots+L_{p}$ is a reduced curve $(i=1,2, \ldots, p)$; hence we have

$$
h^{0}\left(S, \mathscr{O}_{S}\left(\bar{C}_{n}\right)\right)-h^{0}\left(S, \mathscr{O}_{S}\left(\bar{C}_{n}-L_{1}-L_{2}-\cdots-L_{p}\right)\right)=p \text {. }
$$

Remark 2.11. The last proposition shows that for curves on $S$ the bound of Theorem 2.1 is attained in most cases. If $h<k$ then $C_{k}^{\prime}$ has fixed lines, so $\alpha_{k+1}>0$. If $h=k$ we have two possibilities.

(1) $C_{h}^{\prime}$ has $p$ fixed lines (necessarily simple), so we have $\alpha_{h+1}=p$.

(2) $C_{h}^{\prime}$ has no fixed components. In this case $\alpha_{h+1}=0$ and the homogeneous ideal $I(C)$ is generated by $S$ and by $h^{0}\left(S, \mathscr{O}_{S}\left(C_{h}^{\prime}\right)\right)$ polynomials of degree $h$.

Corollary 2.12. Let $C \subset S$ be a curve of type (*) not lying on a quadric.Then $\alpha_{n} \leq 6$ for each $n>h$.

Proof. In fact, using the terminology of Theorem 2.10, we have $\alpha_{h}=p$; now $p \leq 6$ by item (i) of Remark 2.3 .

Remark 2.13. We can see that for curves $C \subset S$ of type $(*)$ generated in degree $h$, the number $\alpha_{h}$ is not bounded. The curve $C_{h}^{\prime}$, linked to $C$ in a complete intersection $(3, h)$ is a curve without fixed components and such that $C_{h}^{\prime}-\pi$ is not an effective divisor on $S$.

Let us take a curve $D$ of type $(n ; n-1,0,0,0,0,0)$ : it has no fixed lines and $D-\pi$, of type $(n-3 ; n-2,-1,-1,-1,-1,-1)$, is not an effective divisor on $S$. Choose $h \geq 2 n, h>2$; let $C$ be of type $(3 h-n ; h-n+$ $1, h, h, h, h, h)$; for this curve we have $C_{h}^{\prime}=D$ and $\alpha_{h}=h^{0}\left(S, \mathscr{O}_{S}\left(C_{h}^{\prime}\right)\right)=$ $2 n+1$. So if we set, e.g., $h=2 n$, we have a curve of degree $4 n-1$ with $\alpha_{h}=2 n+1$.

We shall see in $\S 4$ that for any curve of type $(*)$ one can construct a curve of type $(*)$ which is generated in degree $h$ by $\alpha_{h}$ elements.

Let $C \subset S$ be a curve which is generated in degree $\leq h \quad(h \geq 3)$ and is not ACM; we want to show that the graded Betti numbers of $\mathscr{I}_{C}$ are determined by the fourth differences of the Hilbert function of $C$. 
When $C$ is generated in degree $h$ its linked curve $C_{h}^{\prime}$ has no fixed components, however two possibilities can arise: either $C_{h}^{\prime}$ is of type (*) (i.e., it is linearly equivalent to a reduced and irreducible curve), or $C_{h}^{\prime}$ is not connected and in this case it is composed with a pencil of conics (see below).

If $H^{2}\left(\mathbb{P}^{3}, \mathscr{I}_{C}(h-1)\right) \neq 0$ then, by the speciality theorem, $C$ is the complete intersection of $S$ with a surface of degree $h$ : in this case it is well known that the graded Betti numbers of $\mathscr{I}_{C}$ can be seen in $\Delta^{4} H(C,-)$. Hence we may assume $H^{2}\left(\mathbb{P}^{3}, \mathscr{I}_{C}(h-1)\right)=0$.

Now we want to characterize the curves $C$ such that $C_{h}^{\prime}$ is not connected: this precisely happens when $H^{1}\left(\mathbb{P}^{3}, \mathscr{I}_{C}(h-1)\right) \neq 0$. In fact we know that, for any $n$, the following equality holds [R, §2]:

$$
h^{1}\left(\mathbb{P}^{3}, \mathscr{I}_{C_{h}^{\prime}}(n)\right)=h^{1}\left(\mathbb{P}^{3}, \mathscr{I}_{C}(h-1-n)\right),
$$

and since $C_{h}^{\prime}$ is reduced it follows that: $C_{h}^{\prime}$ is disconnected if and only if $h^{1}\left(\mathbb{P}^{3}, \mathscr{I}_{C}(h-1)\right)>0$, and in this case, $h^{1}\left(\mathbb{P}^{3}, \mathscr{I}_{C}(h-1)\right)=\lambda-1$, where $\lambda=\#$ connected components of $\left.C_{h}^{\prime}\right\}$, and $\lambda>1$.

The components of $C_{h}^{\prime}$ must be $\lambda$ conics of $S$ belonging to the same pencil (see [G, Proposition 2.2 and following]). If, for instance, the pencil of conics is of type $(3 ; 1,1,1,1,1,2)$, then $C_{h}^{\prime}$ must be of type $(3 \lambda ; \lambda, \lambda, \lambda, \lambda, \lambda, 2 \lambda)$ so that $C$ is of type $(3 h-3 \lambda ; h-\lambda, h-\lambda, h-\lambda, h-\lambda, h-\lambda, h-2 \lambda)$, where $\lambda \leq h / 2$ to make $C$ effective. In any case, whatever may be the type of the pencil of conics, we have $d=\operatorname{deg} C=3 h-2 \lambda$, and $h^{1}\left(\mathbb{P}^{3}, \mathscr{I}_{C}(h-1)\right)=\lambda-1$.

Since $H(C, h-1)=3\left(\begin{array}{c}h \\ 2\end{array}\right)+1=P(C, h-1)-\lambda+1$, an easy computation shows that the sequence $\Delta^{4} H(C,-)$ is the following:

\begin{tabular}{ccccccccccccc}
$n$ & 0 & 1 & 2 & 3 & 4 & $\cdots$ & $h-1$ & $h$ & $h+1$ & $h+2$ & $h+3$ & $h+4$ \\
\hline & & & & & & & & & & & \\
$\Delta^{4} H$ & 1 & 0 & 0 & -1 & 0 & $\cdots$ & 0 & $-\lambda-1$ & $\lambda$ & $\lambda$ & $1-\lambda$ & 0
\end{tabular}

Theorem 3.1. Let $C \subset S$ be a curve of type $(*)$ generated in degree $\leq h$, such that $h^{1}\left(\mathbb{P}^{3}, \mathscr{I}_{C}(h-1)\right)=\lambda-1>0$. Then a minimal free resolution for $\mathscr{I}_{C}$ has the form

$$
\begin{aligned}
0 \rightarrow \mathscr{O}(-h-3)^{\oplus(\lambda-1)} \rightarrow \mathscr{O}(-h-2)^{\oplus \lambda} \oplus \mathscr{O}(-h-1)^{\oplus \lambda} \\
\quad \rightarrow \mathscr{O}(-h)^{\oplus(\lambda+1)} \oplus \mathscr{O}(-3) \rightarrow \mathscr{I}_{C} \rightarrow 0 .
\end{aligned}
$$

Proof. The number $\alpha_{h}$ of generators of $I(C)$ is already known by Theorem 2.8. By the minimality of the resolution the first syzygies cannot begin before $h+1$, and the second syzygies cannot begin before $h+2$; since we know the Hilbert function, $\beta_{h+1}$ is known (Lemma 1.2). Now we want to check that in degree $h+2$ there are not second syzygies.

By assumption $\alpha_{h+2}=0$, so we may have $\beta_{h+2}=\lambda+p$ and $\gamma_{h+2}=p$ for some $p \geq 0$. We have to prove that $p=0$, i.e. that the $\lambda$ first syzygies in degree $h+1$ are not related by second syzygies in degree $h+2$. To do this we choose suitable generators for $I(C)$ : by the isomorphism $H^{0}\left(S, \bar{I}_{C}(h)\right) \simeq$ $H^{0}\left(S, \mathscr{O}_{S}\left(C_{h}^{\prime}\right)\right)$, it is enough to choose $\lambda+1$ generators in $H^{0}\left(S, \mathscr{O}_{S}\left(C_{h}^{\prime}\right)\right)$.

Let $\Gamma_{1}, \Gamma_{2}, \ldots, \Gamma_{\lambda}, \Gamma_{1}^{\prime}, \Gamma_{2}^{\prime}, \ldots, \Gamma_{\lambda}^{\prime}$ be $2 \lambda$ distinct conics belonging to the same pencil of the conics of $C_{h}^{\prime}$, and let $H_{1}, H_{2}, \ldots, H_{\lambda}, H_{1}^{\prime}, H_{2}^{\prime}, \ldots, H_{\lambda}^{\prime}$ be 
the planes containing them: denote by $L$ the axis of this pencil. Then the following $\lambda+1$ divisors:

$$
\begin{gathered}
\Gamma_{1}+\Gamma_{2}+\cdots+\Gamma_{\lambda} ; \\
\Gamma_{1}^{\prime}+\Gamma_{2}+\cdots+\Gamma_{\lambda}, \\
\cdots \cdots \cdots+\cdots \cdot \cdots \\
\Gamma_{1}^{\prime}+\Gamma_{2}^{\prime}+\cdots+\Gamma_{\lambda}^{\prime},
\end{gathered}
$$

single out a basis for $H^{0}\left(S, \overline{\mathscr{I}}_{C}(h)\right)$, hence $\lambda+1$ generators of $I(C)$ of degree $h$. In this way we can find $\lambda+1$ surfaces $F_{1}, F_{2}, \ldots, F_{\lambda+1}$ in $\mathbb{P}^{3}$ such that:

$$
\begin{gathered}
F_{1} \cap S=C+\Gamma_{1}+\Gamma_{2}+\cdots+\Gamma_{\lambda}, \\
F_{2} \cap S=C+\Gamma_{1}^{\prime}+\Gamma_{2}+\cdots+\Gamma_{\lambda}, \\
\cdots \cdots \cdots \cdots \cdots \cdots \cdots \cdots \cdots+\cdots \cdots+\Gamma_{1}^{\prime}+\cdots+\Gamma_{\lambda-1}^{\prime}+\Gamma_{\lambda}, \\
F_{\lambda} \cap S=C+\Gamma_{\lambda}, \\
F_{\lambda+1} \cap S=C+\Gamma_{1}^{\prime}+\Gamma_{2}^{\prime}+\cdots+\Gamma_{\lambda}^{\prime} .
\end{gathered}
$$

It is easily seen that the generators of $I(C), F_{1}, F_{2}, \ldots, F_{\lambda+1}, S$ are related by the following independent syzygies:

$$
\begin{gathered}
\left(H_{1}^{\prime},-H_{1}, 0, \ldots, 0,0, G_{1}\right), \\
\left(0, H_{2}^{\prime},-H_{2}, \ldots, 0,0, G_{2}\right), \\
\quad \cdots . . . . . . . . . . . . . \\
\left(0,0,0, \ldots, H_{\lambda}^{\prime},-H_{\lambda}, G_{\lambda}\right),
\end{gathered}
$$

where $G_{1}, G_{2}, \ldots, G_{\lambda}$ are suitable surfaces of degree $h-2$.

These syzygies are not related by second syzygies of degree $h+2$, as one can easily check, so $\gamma_{h+2}=0$ and $\beta_{h+2}=\lambda$. Finally, by Lemma 1.2, item (3), one has $\beta_{h+3}=0$, hence $\gamma_{h+3}=\lambda-1$.

Now consider the case $H^{1}\left(\mathbb{P}^{3}, \mathscr{I}_{C}(h-1)\right)=0$. Since we assumed that in degree $h-1$ there is no speciality, $H(C, h-1)=P(C, h-1)$; so the Hilbert function of $C$ is known and one can compute the sequence $\Delta^{4} H(C,-)$ :

\begin{tabular}{ccccccccccc}
$n$ & $\cdots$ & 3 & 4 & $\cdots$ & $h-2$ & $h-1$ & $h$ & $h+1$ & $h+2$ & $h+3$ \\
\hline$\Delta^{4} H$ & $\cdots$ & -1 & 0 & $\cdots$ & 0 & 0 & $d-3 h$ & $6 h-2 d-3$ & $d-3 h+3$ & 0
\end{tabular}

where, as usual, $d$ is the degree of $C$.

Theorem 3.2. Let $C \subset S$ be a curve of type (*) generated in degree $\leq h$, non$A C M$, and such that $h^{1}\left(\mathbb{P}^{3}, \mathscr{I}_{C}(h-1)\right)=0$. Then a minimal free resolution of $\mathscr{I}_{C}$ has the form:

$$
\begin{aligned}
0 \rightarrow \mathscr{O}(-h-2)^{\oplus(3 h-d-3)} \rightarrow \mathscr{O}(-h-1)^{\oplus(6 h-2 d-3)} \\
\rightarrow \mathscr{O}(-h)^{\oplus(3 h-d)} \oplus \mathscr{O}(-3) \rightarrow \mathscr{I}_{C} \rightarrow 0 .
\end{aligned}
$$

Proof. By the minimality of the resolution we have $\alpha_{h}=3 h-d$, and $\beta_{h+1}=$ $6 h-2 d-3$. Now we apply Lemma 1.2, (3) to conclude that $\gamma_{h+2}=3 h-d-3$, since we know (Lemma 1.4) that $\sigma>\varepsilon$. 
Remark 3.3. Recall that if $C \subset S$ is an ACM curve generated in degree $\leq h$, its resolution is known (see [G1, $\S 3]$ ). For these curves, if we except the complete intersections, we have two possibilities:

(1) Curves linked to a conic. The resolution of these curves may be obtained by Theorem 3.1 for $\lambda=1$.

(2) Curves linked to an ACM cubic curve. In this case $d=3 h-3$, and one can check that for such curves the conclusion of Theorem 3.2 is still true.

In this section we determine the graded Betti numbers of a minimal free resolution of any curve $C \subset S$ of type $(*)$ with $h \geq 3$.

For each $m$ such that $h \leq m \leq k+1$ consider $C_{m}^{\prime}$ and its Zariski decomposition: $C_{m}^{\prime}=s_{1} L_{1}+s_{2} L_{2}+\cdots+s_{p} L_{p}+\bar{C}_{m}$. In the sequel we often use the curves $Y_{m}$ defined in the following way: for each $m$ as above we set

$$
Y_{m}=C+s_{1} L_{1}+s_{2} L_{2}+\cdots+s_{p} L_{p} .
$$

By Lemma 2.2 we have that $Y_{m}$ is a curve of type $(*)$ since each $L_{i}$ is a $\left(s_{i}+m\right)$-secant of $C$ by Proposition 2.6; moreover it is easy to check that for these curves the following facts are true:

$-Y_{m}$ is linked to $\bar{C}_{m}$ in a complete intersection $(3, m)$, so it is generated in degree $\leq m$. Moreover if $m>h$ then $H\left(Y_{m}, n\right)=P\left(Y_{m}, n\right)$ for $n \geq m-1$; hence $\Delta^{4} H\left(Y_{m}, n\right)=0$ for $n \geq m+3$.

-The minimum degree of a surface containing $Y_{m}$ and not containing $S$ is still $h$.

Now we want to compute the Hilbert function of $Y_{m}$. To this end observe that for $n \leq m$ we have $h^{0}\left(S, \mathscr{O}_{S}\left(\left(Y_{m}\right)_{n}^{\prime}\right)\right)=h^{0}\left(S, \mathscr{O}_{S}\left(C_{n}^{\prime}\right)\right)$ because for a complete intersection $n \pi$ containing $C$, since $n \leq m$, we have: $n \pi \sim C+$ $\sum_{i=1}^{p} s_{i} L_{i}+\sum_{i=1}^{t} r_{i} L_{i}+\bar{C}_{n}$, and one sees that the curves

$$
C_{n}^{\prime}=\sum_{i=1}^{p} s_{i} L_{i}+\sum_{i=1}^{t} r_{i} L_{i}+\bar{C}_{n} ; \quad\left(Y_{m}\right)_{n}^{\prime}=\sum_{i=1}^{t} r_{i} L_{i}+\bar{C}_{n},
$$

linked to $C$ and to $Y_{m}$ respectively, differ only in their fixed component. In conclusion we have

$$
\Delta H\left(Y_{m}, n\right)= \begin{cases}\Delta H(C, n) & \text { for } n \leq m, \\ \Delta P\left(Y_{m}, n\right) & \text { for } n \geq m,\end{cases}
$$

moreover $\Delta H\left(Y_{m}, n\right)=\operatorname{deg} Y_{m}$ for $n \geq m$, because $\left(Y_{m}\right)_{m-1}^{\prime}$ is already a reduced curve.

We shall use these curves to determine inductively the graded Betti numbers of the curve $C$ : by knowing the graded Betti numbers of $Y_{m-1}$ and the Hilbert function of $Y_{m}$ we obtain the Betti numbers of $Y_{m}$; in conclusion we describe the resolution of $Y_{k+1}=C$. The first step is a somewhat special situation, so we first consider curves generated in degree $\leq h+1$.

Let $C \subset S$ be a curve of type $(*)$, generated in degree $\leq h+1$. Suppose that $\alpha_{h}=1$ and $\alpha_{h+1}=\lambda$, where $1<\lambda \leq 6$ (if $\lambda=1, C$ is an ACM curve and its resolution is known); in this case $C_{h}^{\prime}$ is the union of $\lambda$ skew lines, and $C_{h+1}^{\prime}$ has no fixed components. By a simple computation one can see that the sequence $\Delta^{4} H(C,-)$ is the following: 


\begin{tabular}{ccccccccccccc}
$n$ & 0 & 1 & 2 & 3 & 4 & $\cdots$ & $h-1$ & $h$ & $h+1$ & $h+2$ & $h+3$ & $h+4$ \\
\hline$\Delta^{4} H$ & 1 & 0 & 0 & -1 & 0 & $\cdots$ & 0 & -1 & $-\lambda$ & $2 \lambda$ & $-\lambda+1$ & 0
\end{tabular}

Proposition 4.1. Let $C \subset S$ be a curve of type $(*)$, generated in degree $\leq h+1$ and such that $\alpha_{h}=1, \alpha_{h+1}=\lambda>1$. Then a minimal free resolution of $\mathscr{I}_{C}$ is of the following kind

$$
\begin{aligned}
0 \rightarrow & \mathscr{O}(-h-3)^{\oplus(\lambda-1)} \rightarrow \mathscr{O}(-h-2)^{\oplus 2 \lambda} \\
& \rightarrow \mathscr{O}(-h-1)^{\oplus \lambda} \oplus \mathscr{O}(-h) \oplus \mathscr{O}(-3) \rightarrow \mathscr{I}_{C} \rightarrow 0 .
\end{aligned}
$$

Proof. One has $\beta_{h+1}=0$ because a complete intersection $(3, h)$ has only one first syzygy of degree $h+3 ; \beta_{h+2}=2 \lambda$ since $\alpha_{h+2}=\gamma_{h+2}=0$; finally, by Lemma 1.2(3), we have $\gamma_{h+3}=\lambda-1$.

Remark 4.2. We want to give a deeper insight into the above situation: in fact it happens that the curve $Y_{h}$ has one first syzygy in degree $h+3$ (the trivial one), but $C$ has no first syzygies in this degree. We shall see later that the trivial syzygy is generated by the $2 \lambda$ syzygies of degree $h+2$.

This fact (a first syzygy which "disappears") can take place only when $\alpha_{h}=1$ : for this reason we want to clarify the geometrical meaning of the situation.

Let $C \subset S$ be a curve of type $(*)$ with $\alpha_{h}=1, \alpha_{h+1}=\lambda$. We may assume that the $\lambda$ components of $C_{h}^{\prime}$ are the exceptional divisors $E_{1}, E_{2}, \ldots, E_{\lambda}[\mathrm{H}$, $\mathrm{V}$, Proposition 4.10]; thus the type of $C$ is

$$
(3 h ; \underbrace{h+1, \ldots, h+1}_{\lambda \text { times }}, h, \ldots, h) .
$$

Further we have

$$
\begin{aligned}
& C_{h}^{\prime}:(\underbrace{0 ;-1, \ldots,-1}_{\lambda \text { times }}, 0, \ldots, 0) ; \quad \bar{C}_{h}:(0 ; 0,0,0, \ldots, 0) ; \\
& C_{h+1}^{\prime}:(\underbrace{3 ; 0, \ldots, 0}_{\lambda \text { times }}, 1, \ldots, 1) .
\end{aligned}
$$

We want to choose $\lambda$ suitable generators of $I(C)$ of degree $h+1:$ it is the same to take a basis of the vector space

$$
\vee=\frac{H^{0}\left(S, \mathscr{O}_{S}\left(C_{h+1}^{\prime}\right)\right)}{\operatorname{Im} \bar{\varphi}_{h+1}}
$$

where $\bar{\varphi}_{h+1}$ has been defined in the proof of Theorem 2.8 .

Consider the following basis of $v$ :

$$
\begin{aligned}
C_{1}+E_{2}+E_{3}+\cdots+E_{\lambda} ; C_{2}+E_{1}+E_{3}+\cdots+E_{\lambda} ; & ; \\
& C_{\lambda}+E_{1}+E_{2}+\cdots+E_{\lambda-1},
\end{aligned}
$$

where $C_{1}, C_{2}, \ldots, C_{\lambda}$ are reduced and irreducible curves of type $(3 ; 0,1,1$, $1,1,1),(3 ; 1,0,1,1,1,1), \ldots,(3 ; 1, \ldots, 0,1, \ldots, 1)$ respectively. 
This basis of $\mathrm{V}$ allows us to find $\lambda$ surfaces $F_{1}, F_{2}, \ldots, F_{\lambda}$, of degree $h+1$, such that

$$
\begin{aligned}
& F_{1} \cap S=C+C_{1}+E_{2}+E_{3}+\cdots+E_{\lambda}, \\
& F_{2} \cap S=C+C_{2}+E_{1}+E_{3}+\cdots+E_{\lambda}, \\
& F_{\lambda} \cap S=C+C_{\lambda}+E_{1}+E_{2}+\cdots+E_{\lambda-1} .
\end{aligned}
$$

If we denote by $G$ the generator of $I(C)$ of degree $h$, a set of minimal generators of $I(C)$ is the following:

$$
S, G, F_{1}, F_{2}, \ldots, F_{\lambda} \text {. }
$$

To find the syzygies among these generators consider (for $i=1,2, \ldots, \lambda$ ) two planes $H_{i}, H_{i}^{\prime}$ in the pencil of axis $E_{i}$ and denote $\Gamma_{i}, \Gamma_{i}^{\prime}$ the conics intersected on $S$ by these planes $\left(\Gamma_{1}\right.$ and $\Gamma_{1}^{\prime}$ are of type $(3 ; 2,1,1,1,1,1)$, and so on). Let us take the quadrics $Q_{i}, Q_{i}^{\prime}$ which meet $S$ in $C_{i}$ and $\Gamma_{i}, C_{i}$ and $\Gamma_{i}^{\prime}$ respectively.

We observe that the surfaces $H_{i} F_{i}$ and $Q_{i} G$ intersect $S$ in the same divisor, namely $C+C_{i}+\Gamma_{i}+E_{1}+\cdots+E_{\lambda}$. So we have the following $2 \lambda$ syzygies:

$$
\begin{aligned}
& S_{i}=\left(-A_{i}, Q_{i}, 0, \ldots,-H_{i}, \ldots, 0\right), \\
& S_{i}^{\prime}=\left(-A_{i}^{\prime}, Q_{i}^{\prime}, 0, \ldots,-H_{i}^{\prime}, \ldots, 0\right) \quad(i=1,2, \ldots, \lambda),
\end{aligned}
$$

where $A_{i}$ and $A_{i}^{\prime}$ are suitable surfaces of degree $h-1$.

From the equalities

$$
Q_{i} G-H_{i} F_{i}=A_{i} S, \quad Q_{i}^{\prime} G-H_{i}^{\prime} F_{i}=A_{i}^{\prime} S,
$$

multiplying the first by $H_{i}^{\prime}$, the second by $H_{i}$, and subtracting, one gets

$$
\left(H_{i}^{\prime} Q_{i}-H_{i} Q_{i}^{\prime}\right) G=\left(H_{i}^{\prime} A_{i}-H_{i} A_{i}^{\prime}\right) S,
$$

so $H_{i}^{\prime} Q_{i}-H_{i} Q_{i}^{\prime}=S, H_{i}^{\prime} A_{i}-H_{i} A_{i}^{\prime}=G$ since $S$ is irreducible and $G$ does not contain $S$. In particular we have obtained the trivial syzygy between $S$ and $G$.

Looking at $S_{i}, S_{i}^{\prime}$ one sees that there are the following $\lambda-1$ second syzygies of degree $h+3$ :

$$
H_{i-1}^{\prime} S_{i-1}-H_{i-1} S_{i-1}^{\prime}-H_{i}^{\prime} S_{i}+H_{i} S_{i}^{\prime}=0 \quad(i=2,3, \ldots, \lambda) .
$$

Finally, we see that these are the only second syzygies of degree $h+3$. In fact if $\sum_{i=1}^{\lambda}\left(L_{i} S_{i}+L_{i}^{\prime} S_{i}^{\prime}\right)=0$, where $L_{i}$ and $L_{i}^{\prime}$ are linear forms, then the $j$ th component $(j=3, \ldots, \lambda+2)$ is $-L_{j} H_{j}-L_{j}^{\prime} H_{j}^{\prime}$, and this vanishes only if $L_{j}=H_{j}^{\prime}, L_{j}^{\prime}=-H_{j}$.

When $C \subset S$ is a curve generated in degree $\leq h+1$ and $\alpha_{h} \geq 2$, we have no remarkable differences with the general case; we insert the next proposition for completeness and for a better clarification of the inductive step.

Proposition 4.3. Let $C \subset S$ be a curve of type $(*)$ generated in degree $\leq h+1$, with $\alpha_{h} \geq 2$. Then the graded Betti numbers of $\mathscr{I}_{C}$ are determined by the resolution of $\mathscr{I}_{Y_{h}}$ and by the sequence $\Delta^{4} H(C,-)$.

Proof. Since $C$ is generated in degree $h+1$ the curve $C_{h}^{\prime}$ is reduced (and has $\alpha_{h+1}$ fixed lines), hence $h=k$; in this case $H(C, n)=P(C, n)$ for $n \geq k$, thus $\Delta^{4} H(C, n)=0$ for $n \geq h+4$. 
According to the different resolutions of $\mathscr{I}_{Y}$ (see $\S 3$ ) there are two possibilities.

(a) $H^{1}\left(\mathbb{P}^{3}, \mathscr{I}_{Y_{h}}(h-1)\right) \neq 0$. By Theorem 3.1 we know $\alpha_{h}^{\prime}, \beta_{h+1}^{\prime}, \beta_{h+2}^{\prime}, \gamma_{h+3}^{\prime}$, the Betti numbers of $Y_{h}$. Recalling Lemma 1.2, for the graded Betti numbers of $\mathscr{I}_{C}$, one has: $\alpha_{h}=\alpha_{h}^{\prime}$ and $\alpha_{h+1}$ is given; $\beta_{h+1}=\beta_{h+1}^{\prime}$ and $\beta_{h+2}=$ $\Delta^{4} H(C, h+2)$. In fact $\alpha_{h+2}=0$ and $\gamma_{h+2}=\gamma_{h+2}^{\prime}$ because (since now) we have only the syzygies $\beta^{\prime}$, and $\gamma_{h+2}^{\prime}=0$ by Theorem 3.1 (see the picture). So to the former $\beta_{h+2}^{\prime}$ first syzygies (relating the $\alpha_{h}$ generators of degree $h$ ) one must add $\beta_{h+2}^{\prime \prime}=\Delta^{4} H(C, h+2)-\beta_{h+2}^{\prime}$ "new" syzygies which link the generators of degree $h+1$. Finally $\gamma_{h+3}=-\Delta^{4} H(C, h+3)$ and, as before, one can distinguish the $\gamma_{h+3}^{\prime \prime}$ "new" second syzygies from the $\gamma_{h+3}^{\prime}$ former ones.

\begin{tabular}{lllllllllll}
$n$ & $\cdots$ & 2 & 3 & 4 & $\cdots$ & $h$ & $h+1$ & & $h+2$ & $h+3$ \\
\hline \#gen. & & & 1 & & & $\alpha_{h}$ & $\alpha_{h+1}$ & & & \\
& & & & & & & & $\beta_{h+2}^{\prime}$ & \\
\#1st syz. & & & & & & $\beta_{h+1}^{\prime}$ & & & \\
& & & & & & & & $\beta_{h+2}^{\prime \prime}$ & \\
& & & & & & & & & \\
& & & & & & & & & &
\end{tabular}

\#2nd syz.

\begin{tabular}{lllllllllll} 
& & & $\gamma_{h+3}^{\prime \prime}$ \\
\hline$\Delta H^{4}(C,-)$ & $\cdots$ & 0 & -1 & 0 & $\cdots$ & $-\alpha_{h}$ & $\beta_{h+1}^{\prime}-\alpha_{h+1}$ & $\beta_{h+2}$ & $-\gamma_{h+3}$
\end{tabular}

(b) $H^{1}\left(\mathbb{P}^{3}, \mathscr{J}_{Y_{h}}(h-1)\right)=0$. By Theorem 3.2 we know $\alpha_{h}^{\prime}, \beta_{h+1}^{\prime}, \gamma_{h+2}^{\prime}$. So $\alpha_{h}=\alpha_{h}^{\prime}, \alpha_{h+1}$ is given; applying again Lemma 1.2 we have $\beta_{h+1}=\beta_{h+1}^{\prime}, \beta_{h+2}=$ $\Delta^{4} H(C, h+2)+\gamma_{h+2}^{\prime}$ and of course $\gamma_{h+2}=\gamma_{h+2}^{\prime}$; finally one gets $\gamma_{h+3}=$ $-\Delta^{4} H(C, h+3)$.

Now the inductive step can be proved without any difficulty.

Theorem 4.4. Let $C \subset S$ be a curve of type (*). For any $n \geq h+1$ the graded Betti numbers of $\mathscr{I}_{Y_{n+1}}$ are determined by the resolution of $\mathscr{I}_{Y_{n}}$ and by the sequence $\Delta^{4} H\left(Y_{n+1},-\right)$.

Proof. By assumption we know $\alpha_{i}^{\prime}, \beta_{i}^{\prime}, \gamma_{i}^{\prime}$, the Betti numbers of $\mathscr{F}_{Y_{n}}$, and that $\Delta^{4} H\left(Y_{n+1}, r\right)=0$ for $r \geq n+4$ (see at the beginning of this section). For the graded Betti numbers $\alpha_{i}, \beta_{i}, \gamma_{i}$ of $\mathscr{I}_{Y_{n+1}}$ one has

$$
\begin{aligned}
& \alpha_{i}=\alpha_{i}^{\prime} \text { for } i \leq n, \text { and } \alpha_{n+1} \text { is given, } \\
& \beta_{i}=\beta_{i}^{\prime} \text { for } i \leq n+1, \text { and } \beta_{n+2}=\Delta^{4} H\left(Y_{n+1}, n+2\right)+\gamma_{n+2}^{\prime}, \\
& \gamma_{i}=\gamma_{i}^{\prime} \text { for } i \leq n+2, \text { and } \gamma_{n+3}=-\Delta^{4} H\left(Y_{n+1}, n+3\right) .
\end{aligned}
$$

Remark 4.5. Summing up the results of the last two sections we may observe the following relevant facts about the graded Betti numbers of a curve $C \subset S$ of type $(*)$.

(1) The following fact is true in general (i.e., for any $n \geq h$ if $\bar{C}_{h}$ is not composed with a pencil and $\alpha_{h}>1$; for any $n \geq h+1$ if $\alpha_{h}=1$; for any $n>h+3$ if $\bar{C}_{h}$ is composed with a pencil): the graded Betti numbers 
of $Y_{n+1}$ are obtained from those of $Y_{n}$ just adding $\alpha_{n+1}$ "new" generators, $\beta_{n+2}=\Delta^{4} H\left(Y_{n+1}, n+1\right)+\gamma_{n+2}$ "new" first syzygies which link the "new" $\alpha_{n+1}$ generators to the others, and $\gamma_{n+3}=-\Delta^{4} H\left(Y_{n+1}, n+3\right)$ "new" second syzygies which link the "new" $\beta_{n+2}$ syzygies to the others (see Theorem 4.4).

The cases that are left out have been explicitly studied in this section.

(2) For any $n \geq h$ consider the graded Betti numbers of $Y_{n}$, say $\alpha_{i}^{\prime}, \beta_{i}^{\prime}, \gamma_{i}^{\prime}$. If $\gamma_{n+2}^{\prime}>0$ then $\beta_{n+2}^{\prime}=0$; if $\gamma_{n+2}^{\prime}=0$ then either $Y_{n}$ is an ACM curve or $\beta_{n+2}^{\prime}>0$ and $\gamma_{n+3}^{\prime}>0$ (see Theorem 3.1).

Example 4.6. We want to illustrate how one constructs the resolution of a curve on $S$.

Consider the curve $C:(15 ; 8,7,4,4,2,2)$. It has degree $d=18$ and genus $g=28$. For $C$ we have $h=9, k=10$, and $\Delta H(C,-)$ is the following:

\begin{tabular}{lllllllllllll}
$n$ & $\cdots$ & 2 & 3 & 4 & 5 & 6 & 7 & 8 & 9 & 10 & 11 & 12 \\
\hline & & & & & & & & & & & & \\
$\Delta H(C,-)$ & $\cdots$ & 6 & 9 & 12 & 15 & 18 & 21 & 24 & 24 & 20 & 18 & 18
\end{tabular}

We have $C_{9}^{\prime}:(12 ; 1,2,5,5,7,7)=2(1 ; 0,0,0,0,1,1)+2(2 ; 0,1,1$, $1,1,1)+(2 ; 1,0,1,1,1,1)+(4 ; 0,0,2,2,2,2)$; so $C_{9}^{\prime}$ has three fixed lines with multiplicities $2,2,1$, and $\bar{C}_{9}:(4 ; 0,0,2,2,2,2)$ is union of two conics of the same pencil (note that we are not in the general case of Remark 4.5(i). Thus $\alpha_{9}=3, \alpha_{10}=3$, and $\alpha_{11}=2$ (see Theorems 2.8 and 2.10).

$Y_{9}$ is of type $(23 ; 9,9,7,7,7,7)$; by Theorem 3.1 we get for this curve the following Betti numbers: $\alpha_{9}=3, \beta_{10}=2, \beta_{11}=2, \gamma_{12}=1$ (see in the picture below the underlined numbers).

$Y_{10}$ is of type $(18 ; 8,8,5,5,4,4)$; looking at the Hilbert function of $C$ we get

\begin{tabular}{lllllllllllllll}
$n$ & 0 & 1 & 2 & 3 & 4 & 5 & 6 & 7 & 8 & 9 & 10 & 11 & 12 & 13 \\
\hline
\end{tabular}

$\begin{array}{llllllllllllllll}\Delta^{4} H\left(Y_{10},-\right) & 1 & 0 & 0 & -1 & 0 & 0 & 0 & 0 & 0 & -3 & -1 & 8 & -4 & 0\end{array}$

and by comparison with the Betti numbers of $Y_{9}$ (Theorem 4.3) we obtain $\beta_{11}=6, \gamma_{12}=3$ (see the barred numbers).

$Y_{11}=C:$ as before we can calculate $\Delta^{4} H(C,-)$

\begin{tabular}{lllllllllllll}
$n$ & $\cdots$ & 3 & 4 & 5 & 6 & 7 & 8 & 9 & 10 & 11 & 12 & 13 \\
\hline$\Delta^{4} H(C,-)$ & $\cdots$ & -1 & 0 & 0 & 0 & 0 & 0 & -3 & -1 & 6 & 0 & -2
\end{tabular}

and comparing with the previous numbers we have $\beta_{12}=4, \gamma_{13}=2$ (numbers with the hats).

Summing all we have the following graded Betti numbers: 


\begin{tabular}{lccccccccccc}
$n$ & 0 & 1 & 2 & 3 & $\cdots$ & 9 & 10 & 11 & 12 & 13 & $\cdots$ \\
\hline \# gen. & & & 1 & & $\underline{3}$ & $\overline{3}$ & $\hat{2}$ & & & \\
\#1 st syz. & & & & & & & $\underline{2}$ & $\underline{2}+\overline{6}$ & $\hat{4}$ & & \\
\#2nd syz. & & & & & & & & $\underline{1}+\overline{3}$ & $\hat{2}$ &
\end{tabular}

Remark 4.7. In this section we have found an algorithm to determine the graded Betti numbers of any curve $C \subset S$ of type $(*)$, based on the curves $Y_{m}$. Now we want to explain another algorithm which achieves the same result, perhaps in a simpler way.

Given a curve $C$ of type $(*)$ which is not a complete intersection compute first the numbers $h$ and $k$ (see Definition 1.1 and Remark 2.5), and the numbers $\alpha_{n}$ of generators for each $n=h, h+1, \ldots, k+1$. Then, for any $n$ such that $h \leq n \leq k+3$, compute $h^{0}\left(S, \mathscr{O}_{S}\left(\bar{C}_{n-3}+3 \pi\right)\right), h^{0}\left(S, \mathscr{O}_{S}\left(\bar{C}_{n-2}+2 \pi\right)\right)$, $h^{0}\left(S, \mathscr{O}_{S}\left(\bar{C}_{n-1}+\pi\right)\right)$, assuming $h^{0}\left(S, \mathscr{O}_{S}\left(\bar{C}_{n-i}+i \pi\right)\right)=0$ whenever $\bar{C}_{n-i}$ is not an effective divisor on $S(i=1,2,3)$, i.e., when $n-i<h$.

Consider the numbers:

$$
\begin{gathered}
B_{n}=4 \alpha_{n-1}-h^{0}\left(S, \mathscr{O}_{S}\left(\bar{C}_{n-1}+\pi\right)\right)+h^{0}\left(S, \mathscr{O}_{S}\left(\bar{C}_{n-2}+2 \pi\right)\right), \\
G_{n}=4 \beta_{n-1}-\left[10 \alpha_{n-2}-h^{0}\left(S, \mathscr{O}_{S}\left(\bar{C}_{n-2}+2 \pi\right)\right)+h^{0}\left(S, \mathscr{O}_{S}\left(\bar{C}_{n-3}+3 \pi\right)\right)\right] .
\end{gathered}
$$

Three cases can occur:

(1) $\bar{C}_{h}$ is not composed with a pencil and $\alpha_{h}>1$. In this case we have, for each $n$ :

$$
\beta_{n}=B_{n} \quad \text { and } \quad \gamma_{n}=G_{n} .
$$

(2) $\bar{C}_{h}$ is composed with a pencil, say $\bar{C}_{h}=\lambda \Gamma$, where $\Gamma$ is a conic on $S$ and $\lambda>1$. In this case we have

$$
\begin{array}{lll}
\beta_{h+1}=\lambda, & & \\
\beta_{h+2}=\lambda+B_{h+2}, & \gamma_{h+2}=0, & \\
\beta_{h+3}=B_{h+3}, & \gamma_{h+3}=\lambda-1+G_{h+3}, & \\
\beta_{n}=B_{n}, & \gamma_{n}=G_{n}, & \text { for } n>h+3 .
\end{array}
$$

(3) $\alpha_{h}=1$. In this case $\beta_{h+1}, \beta_{h+2}, \gamma_{h+2}, \gamma_{h+3}$ are given in Proposition 4.1; $\beta_{n}=B_{n}$ and $\gamma_{n+1}=G_{n+1}$ for $n \geq h+3$.

The formula for $B_{n}$ is based on Remark 4.5(1), and on Lemma 2.7: in fact the number $B_{n}$ is the dimension of the kernel of the map

$$
\mathrm{V} \otimes H^{0}\left(S, \mathscr{O}_{S}(1)\right) \rightarrow \mathrm{W},
$$

where $\mathrm{V}$ is the quotient of $H^{0}\left(S, \mathscr{O}_{S}\left(\bar{C}_{n-1}\right)\right)$ modulo $H^{0}\left(S, \mathscr{O}_{S}\left(\bar{C}_{n-2}+\pi\right)\right)$ and $\mathrm{W}$ is the quotient of $H^{0}\left(S, \mathscr{O}_{S}\left(\bar{C}_{n-1}+\pi\right)\right)$ modulo $H^{0}\left(S, \mathscr{O}_{S}\left(\bar{C}_{n-2}+2 \pi\right)\right)$. Observe that this map is surjective since it is induced by the cup-product morphism on $\bar{C}_{n-1}$.

The formula for $G_{n}$ can be obtained with a similar argument.

Finally we consider curves lying on a smooth quadric; this case is very simple, since, as we now see, the graded Betti numbers of such curves only depend on their Hilbert function. 
Theorem 4.8. Let $C \subset \mathbb{P}^{3}$ be a curve contained on a smooth quadric; if $C$ is of type $(a, b)$, with $0<a<b-1$, then a minimal free resolution of $\mathscr{I}_{C}$ has the following form:

$$
\begin{aligned}
0 & \rightarrow \mathscr{O}(-b-2)^{\oplus(b-a-1)} \rightarrow \mathscr{O}(-b-1)^{\oplus(2 b-2 a)} \\
& \rightarrow \mathscr{O}_{\mathbb{P}^{3}}(-b)^{\oplus(b-a+1)} \oplus \mathscr{O}(-2) \rightarrow \mathscr{I}_{C} \rightarrow 0 .
\end{aligned}
$$

Proof. Recall that if $b=a$ or $b=a+1$, then $C$ is an ACM curve and its resolution is known. Computing the Hilbert function of $C$ (see $[\mathrm{G} 1, \S 6])$ we have the following sequence $\Delta^{4} H(C,-)$ :

\begin{tabular}{ccccccccccr}
$n$ & 0 & 1 & 2 & 3 & $\cdots$ & $b-1$ & $b$ & $b+1$ & $b+2$ & $b+3$ \\
\hline$\Delta H^{4}$ & 1 & 0 & -1 & 0 & $\cdots$ & 0 & $a-b-1$ & $2 b-2 a$ & $a+1-b$ & 0
\end{tabular}

hence the result follows by Lemma 1.2.

\section{REFERENCES}

[C] G. Campanella, Standard bases of perfect homogeneous polynomial ideals of height 2, J. Algebra 101 (1986), 47-60.

[E] G. Ellingsrud, Sur le schéma de Hilbert des variétés de codimension 2 dans $\mathbb{P}^{e}$ à cône de Cohen-Macaulay, Ann. Sci. École Norm. Sup. 8 (1975), 423-431.

[G] S. Giuffrida, Hilbert function of a 0-cycle in $\mathbb{P}^{2}$, Matematiche 40 (1985).

[G1] _ The Hilbert function of a curve lying on a smooth cubic surface, Ann. Mat. Pura Appl. (4) 153 (1988), 275-292.

[GeMi1] A. V. Geramita and J. C. Migliore, Hyperplane sections of a smooth curve in $\mathbb{P}^{3}$, Queen's Papers in Pure and Appl. Math., vol. 80, Queen's Univ., Kingston, 1988.

[GeMi2] __, Generators for the ideal of an arithmetically Buchsbaum curve, J. Pure Appl. Algebra 58 (1989), 147-167.

[GM] S. Giuffrida and R. Maggioni, On the Rao module of a curve lying on a smooth cubic surface in $\mathbb{P}^{3}$, Comm. Algebra 18 (1990), 2039-2061.

[GP] L. Gruson and C. Peskine, Genre des courbes de l'espace projectif, Lecture Notes in Math., vol. 687 , Springer, 1978 , pp. 31-59.

[H] R. Hartshorne, Algebraic geometry, Springer-Verlag, New York, 1977.

[H1] _ On the classification of algebraic space curves, Vector Bundles and Differential Equations (Nice, 1979), Birkhäuser, Basel, 1980, pp. 83-112.

[Ha] B. Harbourne, Complete linear systems on rational surfaces, Trans. Amer. Math. Soc. 289 (1985), 213-226.

[HE] J. Harris and D. Eisenbud, Curves in projective space, Les Presses de l'Université de Montréal, Montréal, 1982.

[M] M. Mumford, Curves on algebraic surfaces, Ann. of Math. Stud., no. 59, Princeton Univ. Press, Princeton, N.J., 1966.

[MR] R. Maggioni and A. Ragusa, On the cohomology groups of linked schemes, Boll. Un. Mat. Ital. 2A (1988), 269-276.

[MR1] _ The Hilbert function of generic plane sections of curves of $\mathbb{P}^{3}$, Invent. Math. 91 (1988), 253-258.

[P] G. Pareschi, Curve aritmeticamente Buchsbaum su superfici di grado 3 et 4 dello spazio proiettivo, Ann. Univ. Ferrara Sez. VII 33 (1987), 219-235. 
[PS] C. Peskine and L. Szpiro, Liaison des variétés algébriques I, Invent. Math. 26 (1974), 271302.

[R] A. P. Rao, Liaison among curves in $\mathbb{P}^{3}$, Invent. Math. 50 (1979), 205-217.

[S] T. Sauer, Smoothing projective Cohen-Macaulay space curves, Math. Ann. 272 (1985), 8390.

[W] M. Watanabe, On projective normality of space curves on a nonsingular cubic surface in $\mathbb{P}^{3}$, Tokyo J. Math. 4 (1981), 331-341.

[Z] O. Zariski, The theorem of Riemann-Roch for high multiples of an effective divisor on an algebraic surface, Ann. of Math. 76 (1962), 560-615.

Dipartimento di Matematica, Università di Catania, Viale A. Doria, 6, 95125 Catania, ITALY 